\title{
OFERTA ALIMENTARIA: LOS CONSUMIDORES Y SUS EXPECTATIVAS*
}

\section{Louise Sylvan**}

Resumen: En un siglo de progresos significativos en salud para una gran parte del mundo, lo último que los consumidores podrían haber esperado al finalizar el milenio es que los antiguos problemas de seguridad alimentaria se vieran intensificados por la introducción de nuevos métodos y de la aplicación de nuevas tecnologías en la agroindustria. Los consumidores y los ciudadanos en general han reaccionado con indignación por el riesgo al que han sido expuestos por el gobierno y los expertos. Aunque presentadas como opciones con base estrictamente científica, muchas de las decisiones relativas a la seguridad alimentaria y al comercio de alimentos a niveles nacionales o internacionales contienen un significativo elemento de interés ético, muchas veces no identificado o ignorado. Palabras clave: nocividad, regulación del consumo alimenticio, Asociaciones de Consumidores, Política, Democracia.

\section{FOOD SUPPLY: CONSUMERS AND THEIR EXPECTATIONS}

Abstract: In a century of significant improvements in health for many in the world, the last thing consumers expected at the close of this millennium was that the old problems of food safety would be intensified due to the introduction of new methods and technologies in the agri-business domain. Consumers and citizens have often reacted angrily at the risk that governments and experts have been willing to expose them to. While presented as decisions based solely on science, many of the decisions taken about food safety and food trade, at national and international levels, have a significant ethical element - most of the times unidentified and/or ignored. Keywords: harmfulness, food consumption regulation, Consumer Associations, politics, Democracy.

\section{OFERTA ALIMENTAR: CONSUMIDORES E SUAS EXPECTATIVAS}

Resumo: Em um século de significativos progressos para a saúde de uma grande parte do mundo, o que menos poderiam os consumidores esperar ao finalizar o milênio era o fato de que antigos problemas de segurança alimentar sofressem acentuação como resultado da introdução de novas tecnologias agro-industriais. Os consumidores e os cidadãos, de um modo geral, reagiram com indignação ante o risco que são expostos por iniciativa do governo e especialistas. Ainda que apresentadas como opções viáveis e calcadas em base estritamente científicas, muitas das decisões relativas à segurança alimentar e ao comércio de alimentos em níveis nacionais e/ou internacionais contém significativos elementos de interesse ético, muitas vezes não identificados ou, até mesmo, ignorados.

Palavras chaves: nocividade, regulação de consumo alimentar, Associações de Consumidores, Política, Democracia.

\footnotetext{
* Traducido del Inglés por Steffan Larenas. Oficina Regional para América Latina y el Caribe de Consumers International.

** Presidenta Consumers International. Directora Ejecutiva, Australian Consumers'Association

Correspondencia: E-mail: LSYLVAN@CHOICE.COM.AU
} 


\section{Introducción}

Las expectativas que se hacen los consumidores respecto a la oferta alimentaria no pueden sorprender a nadie que haya pensado un poco el asunto. Aparte de su accesibilidad, los consumidores también suponen que lo que compran debe ser seguro y nutritivo. Muchos consumidores agregarían, además, que les interesa que los métodos de producción sean seguros para la salud humana y el medioambiente. Estas son afirmaciones simples y son afirmaciones éticas donde subyace un sinnúmero creciente de preocupaciones por el incremento de la influencia de los grandes intereses comerciales, muchos de los cuales son a escala global.

Del mismo modo también son bastante sencillas las expectativas de los consumidores en cuanto a cómo sus gobiernos (así como las respectivas agencias internacionales) debieran comportarse en el manejo de las normas y de los estándares de los alimentos, incluso bajo la presión de intereses comerciales.

Aun sin expresarlo con las mismas palabras los consumidores manifiestan su interés en que el gobierno regule apropiadamente en beneficio de los ciudadanos, para asegurar así un alimento seguro, que sean estrictos y vigilantes en el reforzamiento de las leyes, y que sean cautos frente a la introducción de nuevas tecnologías aún no probadas en la elaboración de alimentos. Después de todo, es para la seguridad, la salud pública y la protección de los consumidores que los gobiernos han legislado sobre seguridad alimentaria ${ }^{\mathrm{i}}$.

En los últimos veinte años, los consumidores organizados que defienden sus intereses se han sentido algo desilusionados con sus gobiernos (así como con las agencias internacionales) y con los compromisos que aquéllos han suscrito. Algunos gobiernos han sido mejores que otros y algunas discusiones mejor que otras en los distintos foros internacionales. Sin embargo, existe una gran sensación de que los principios han sido abandonados en favor de los intereses comerciales.

\section{Precaución}

El punto principal para poder mejorar el comportamiento de los gobiernos y de las agencias internacionales consiste en la capacidad de poner en contexto los parámetros de las decisiones políticas. Brevemente, la posición del consumidor antes mencionada sugiere que la postura ética de los gobiernos y agencias internacionales en sus deliberaciones sobre normas alimentarias debiera ser "precautoria". Desde una perspectiva moral, la conducta "precautoria" debiera ser observada tanto en relación con los seres humanos como con respecto al medioambiente.

En el nivel más simple, el principio de precaución dice que la política pública moralmente correcta donde exista incertidumbre sobre los efectos que pueda tener determinado proceso o producto - ya sea para la salud humana o para el medio ambienteconsistiría en demorar la aprobación de cualquier nuevo proceso o tecnología hasta que haya más certidumbre disponible, con el fin de eliminar o disminuir el peligro a un mínimo y como expresión de una posición precautoria.

El principio precautorio ha provocado grandes debates académicos y ha tenido tanto buena como mala prensa. Mucho se ha discutido sobre la búsqueda de definiciones aceptables. Gran parte de la discusión se ha centrado en la distinción entre precaución y evaluación de riesgo. La posición del consumidor, en general, sostiene que la evaluación de riesgo es una herramienta técnica que necesita ser situada dentro de un marco más

\footnotetext{
i Mientras esto es cierto en la mayoría de los casos, uno debe reconocer que no toda la legislación sobre alimentos ha sido dirigida hacia la protección de la población. Algunos países han creado leyes alimentarias con el fin de proteger a la industria y, en algunos casos, como una protección no tarifaria contra las importaciones.
} 
amplio de toma de decisiones, dentro del cual el principio de precaución oriente la recoleccion de información y la selección de las herramientas de evaluación y de las políticas preventivas. En otras palabras, la consideración que el principio de precaución es un principio general y no un derivado de la evaluación de riesgo ${ }^{i \text { i }}$.

Para el consumidor queda claro, después de medio siglo de experiencia con las políticas de salud y medioambiente, que es necesario un fuerte y claro enfoque precautorio y preventivo. Lisa y llanamente se han cometido demasiados errrores por la carencia de estos principios. Cambiar todo esto significa no sólo un cambio de conducta y de actitud de parte de los gobiernos, sino también una revisión de los roles de la ciencia y de la política en la salud medioambiental y en el comercio internacional $^{\text {iii. }}$. El punto es, entonces, asegurar que la toma de decisión refleje mejor la necesidad de prevención y dé cuenta de las limitaciones de que adolece nuestro conocimiento.

La oposición más significativa contra el principio de precaución viene desde la perspectiva industrial, la que, básicamente, argumenta que "el comercio no debe ser ralentizado mientras se demuestre que todo es seguro" o, a un nivel más sofisticado, "que no hay razón para que un producto o proceso sea

ii Véase, por ejemplo, la postura del consumidor y las bases para las conclusiones políticas en los papers que se encuentran disponibles en: www.tacd.org

iii Los "triunfos " del comercio sobre las preocupaciones de la salud están siendo sometidos a un creciente escrutinio no sólo en el debate alimentario. El exitoso desafío moral de los países en desarrollo respecto de las internacionales farmacéuticas, cuestionando la posición de éstas en lo que respecta a los derechos de propiedad intelectual se inclina por el acceso. Esto apareció muy claramente durante la reunión de la Organización Mundial de Comercio en Doha en noviembre del 2001, acerca de que la propiedad intelectual no tiene prioridad sobre el acceso a los medicamentos. Es el primer capítulo de muchas batallas éticas. restringido hasta que no se haya demostrado que no es peligroso" - esencialmente lo contrario del onus probandi y una posición básicamente anti-precautoria. Extremando las cosas, se argumenta que las naciones no tienen derecho a proteger a sus ciudadanos a menos que se demuestre concluyentemente el riesgo y que el costo que implica demostrar las fallas de seguridad no recaiga sobre los que proponen la tecnología sino sobre el Estado que busca la protección ciudadana. Desde el punto de vista del consumidor, ésta es una postura antiética que sólo llevará a resultados trágicos e irreversibles para la gente y el medioambiente.

\section{"Vacas locas"}

Los consumidores se preguntan cómo pudo ocurrir el BSE (encefalopatía espongiforme bovina). Para responder la pregunta de manera elemental podemos decir que la agroindustria decidió ignorar siglos de experiencia que dice que los herbívoros deben ser exactamente eso. Así es que alimentar herbívoros con productos derivados de la carne (que consiste en despojos de animales procesados) puede ser bueno para la agricultura desde la perspectiva de fondo del negocio de la agroindustria, pero puede ser una actividad peligrosa. Nadie lo sabía. La industria entonces adoptó su postura habitual de que al no existir evidencia de riesgo, no hay razón para desaprobar dicha práctica. Pero, como ha ocurrido demasiado frecuentemente en la agroindustria en los últimos 50 años, las cuestiones críticas muy rara vez encuentran respuesta oportunamente y, cuando son respondidas por investigadores independientes (generalmente del ámbito académico), estas personas son a veces ridiculizadas y sus carreras arruinadas, especialmente porque la industria es un sostenedor principal de la así llamada investigación independiente en el sistema universitario.

¿Cómo pudo escaparse tanto de las manos la BSE? Esta pregunta es más complicada y llega al corazón de la posición 
del consumidor mencionada anteriormente respecto a cómo deben proceder los gobiernos. La posición del consumidor mantiene que los gobiernos tienen una exigencia ética de comportarse con precaución para proteger a los ciudadanos. En lugar de eso, en el caso de BSE, el gobierno británico había estructurado una agencia reguladora en la que existía un tremendo conflicto de intereses. El Ministerio de Agricultura, Alimentación y Recursos Forestales, MAFF, no era sólo el responsable de la seguridad alimentaria, sino que también responsable de la promoción tanto de la industria agrícola como de la forestal. El MAFF no podía, por tanto, conducirse de manera completamente precautoria con semejantes condiciones estructurales.

Cuando se llegó a un serio conflicto de intereses con la aparición de la BSE, la entidad fracasó en la protección pública porque temía dañar la industria agrícola, por lo que la BSE no pudo ser contenida a tiempo como debería haber sido.

Inevitablemente, el MAFF le falló también a la industria cuando los consumidores decidieron que el gobierno estaba mintiendo y le dieron la espalda a un producto considerado no seguro. No fue un resultado feliz para nadie, ni una situación que pueda ser remediada completamente; ella ha empeorado, puesto que la enfermedad se ha propagado al extranjero.

La BSE es un clásico ejemplo de un gobierno defraudando la fe pública en cuanto a su primera responsabilidad ética con todos sus ciudadanos y comprometiendo su deber porque su interés está focalizado en sólo un segmento de la población, de una sola industria, quienes son definidos como sus "stakeholders".

Un pequeño grupo, como es la industria ganadera, tiene mucha más influencia que una masa amorfa de ciudadanos pobremente organizados. Nadie argumenta que las necesidades de la industria no deben ser tomadas en cuenta, o que los gobiernos procedan sin la debida consideración de las partes. Pero los gobiernos son, después de todo, gobiernos de toda la ciudadanía, y no pertenecen al sector industrial que "grita más fuerte". La lamentable caída de respeto con que son vistos los políticos en el mundo democrático hoy en día, independientemente de su orientación política, puede ser en parte un indicador de que la gente entiende que aquellos que tienen la misión de gobernar en representación nuestra, a menudo hacen lo contrario, ya sea movidos por la corrupción o por razones ideológicas o, simplemente, por su ineptitud para mantener claras las prioridades.

\section{Alimentos genéticamente modificados}

Mientras que la debacle de la BSE fue un ejemplo de un organismo regulador en conflicto de intereses, que toma malas decisiones como resultado de ese conflicto, quizás sea el caso de los alimentos GM el mejor ejemplo en la última década de que un interés comercial sobrepasa el interés de los ciudadanos.

Esta tecnología, creada principalmente por multinacionales, ha generado innumerables disputas entre consumidores y promotores, entre agencias de las Naciones Unidas, entre los gobiernos -especialmente los EEUU y la Unión Europea - y entre países desarrollados y en vías de desarrollo.

Existen muchas cuestiones morales y éticas en juego respecto a la tecnología genética, tales como: ¿La vida puede llegar a ser una propiedad comercial y puede patentarse? ¿Podemos crear organismos transgénicos, particularmente aquéllos que contienen ADN humano y animal? ¿Quién defiende a la naturaleza? ¿Cómo deberían las naciones asegurarse de que los procesos de toma de decisiones respeten la diversidad cultural, moral y religiosa en nuestra sociedad multicultural? ${ }^{\text {i v }}$ Todas estas cuestiones, y otras más, son dejadas

iv Esas fueron un conjunto de preguntas críticas que surgieron en la Conferencia Australiana de Consenso cuyo tema era la Tecnología Genética, especialmente en lo alimentario. Consultar en:

www.amonline.net.au/consensus/09.htm. 
de lado mientras los gobiernos se apresuran a asegurarse de no quedar atrás en la carrera de la ingeniería genética.

Como siempre, tratándose de una novedad tecnológica, la innovación se plantea de dos maneras. Primero, la tecnología se presenta como esencialmente benigna $\mathrm{y}$, por tanto, libre de ser objeto de un escrutinio especial. En el caso de los alimentos GM, frecuentemente se afirmó que la modificación genética no era sino una aceleración y una versión más precisa de técnicas de selección genética "convencionales" utilizadas por siglos en agronomía y ganadería. Pero el fundamento científico de tales afirmaciones es muy mañoso ya que la humanidad nunca poseyó antes la habilidad para introducir material genético de una especie en una especie diferente y es precisamente esto lo que hace que los OGM sean completamente diferentes a la evolución previa en la producción de alimentos. El hecho de que las compañías multinacionales hayan buscado enérgicamente proteger su propiedad intelectual en términos de innovaciones de OGM, sugiere que están perfectamente conscientes de que existe una diferencia significativa ya que, por definición, las patentes son adjudicadas sólo en tales circunstancias.

La segunda presentación que la industria hace de la innovación tecnológica es bajo el aspecto de la "gran solución" a uno de los grandes problemas mundiales, en este caso, la esperanza de resolver el grave problema del hambre, la desnutrición, y la sustentabilidad ambiental. Con semejante toque de marketing uno pensaría que todos los consumidores del mundo estarían agradecidos, pero no es así. Aquellos de nosotros, lo suficientemente mayores, podemos recordar la promesa de la "Revolución Verde" en la que se usó el mismo lenguaje para promover el uso de pesticidas y nuevas cepas de semillas, a menudo patentadas por multinacionales.

Debido a los efectos insostenibles de las monoculturas y otras dificultades, la "Revolución Verde" creó tantos problemas como los que resolvió. A pesar de esa experiencia, algunos actores influyentes en la escena mundial, además de gobiernos y multinacionales, han tomado la antorcha de la biotecnología con el mismo tono mesiánico, como se puede constatar en el informe del PNUD ( Programa de las Naciones Unidas para el Desarrollo).

A pesar de todo el ruido, el persistente desinterés por parte de los consumidores por la biotecnología y su aplicación en los alimentos, tiene dos raíces. Mucha gente siente que no se les ha dado alternativas apropiadas y que la tecnología les ha sido impuesta. En cartas a los periódicos algunos consumidores expresan que se sienten usados como conejillos de indias por la industria con la complicidad de sus gobiernos.

En un mundo dominado por el capitalismo y los "mercados libres", se dice que el consumidor es el "rey" y que su derecho a escoger en el mercado de oferta es sagrado. Pero en muchos países la industria se ha asegurado que los alimentos GM no sean etiquetados o que la legislación sobre etiquetas sea debilitada. Como resultado, al consumidor se le niega su derecho a elección libre. Él quiere ejercer su derecho a elección por dos razones: una, porque pueden existir dudas acerca de la seguridad de tales productos y preferiría evitarlos; la otra se refiere a puntos de vista de ética personal, ya sea con relación a una preocupación por el medioambiente o una motivación religiosa por los GM (por ejemplo, si se usa el gen de un cerdo o de una vaca en un vegetal, algunos fieles de ciertas religiones querrían saber más para evitar así consumir semejante producto).

Cuando los consumidores escuchan la voz de la industria asegurando que "los consumidores no quieren que se etiqueten productos GM", inevitablemente aquella fuerte postura de la industria es sospechosa ${ }^{\mathrm{v}}$. Después de todo, ¿si los alimentos GM son tan maravillosos como alega la industria, no debieran etiquetarlos de buena gana, obteniendo de esta forma una ventaja en la comercialización de sus productos que porten tal identificación? Cuando la industria y 
algunos gobiernos argumentan que los alimentos GM son esencialmente "lo mismo" pero, al mismo tiempo, se apresuran a patentarlos, lo que los hace aparecer como algo diferente, los consumidores sienten que son tomados por tontos.

Un aspecto de gran significado para los defensores de los consumidores es que el gobierno del mercado más poderoso del mundo, los EEUU, se alineó con la industria en este asunto. Si uno prefiere una interpretación más siniestra, dicho gobierno vio la posición antietiquetado como perjudicial para los intereses de las compañías transnacionales en el comercio mundial.

Los EEUU han mantenido su política anti-etiquetado a pesar de toda la evidencia derivada de la investigación sobre la tendencia de los ciudadanos en el sentido contrario ${ }^{\text {vi }}$. El Gobierno Federal Australiano también se alineó con su industria, aunque se encontró impotente para enrolar al menos ingenuo de los Ministerios de Salud: la ley australiana exigirá un etiquetado razonablemente estricto tal como el de la Unión Europea y Japón.

¿Por qué es importante un correcto rotulaje de los alimentos GM? Es respuesta suficiente el derecho de los ciudadanos a ejercer su poder en el mercado, y más del $90 \%$ de los consumidores -según encuestas realizadas por todo el mundo- han indicado claramente a sus gobiernos que ellos desean contar con etiquetas claras respecto de los GM ( y no seudoetiquetas).

Menos obvio resulta que, a pesar del esfuerzo de los gobiernos por tranquilizar a los ciudadanos, los consumidores se siguen

v A pesar de una supuesta orientación del mercado, las corporaciones alegan contra los intereses de los consumidores, no al nivel de la retórica general, sino al nivel de roles específicos. Se recuerda que la industria hace menos de veinte años afirmó que los consumidores no querían que los ingredientes estuvieran declarados en las etiquetas ¡que eso sería aparentemente confuso para ellos! sintiendo inseguros con respecto a la seguridad de los GM y al aumento de los cultivos GM. Su preocupación puede estar muy bien fundada. En este segundo aspecto los consumidores están señalando claramente que desean actuar con precaución, incluso si sus gobiernos han fracasado en hacerlo.

En las últimas décadas, el etiquetado ha sido muy eficiente en ayudar a los consumidores a elegir alimentos más sanos, existiendo muchos que exigen un etiquetado adecuado para proteger su salud. Por ejemplo, cualquier alimento GM que contenga una toxina o alérgeno conocido en cualquiera de sus formas debe proveer una advertencia nítida en su etiqueta sobre cuáles productos han sido empleados en la producción de ese alimento en particular.

Algunas otras prácticas también han despertado temores. La práctica habitual de realizar modificaciones genéticas usando marcadores genéticos resistentes a los antibióticos -algo que va a ser abandonado luego de la reunión organizada por la OCDE (Organización de Cooperación y Desarrollo Económicos) en la materia- es un ejemplo. Si ocurriera que tal resistencia se extendiera a una bacteria patógena a través de un alimento OGM, podría causar un gran daño. Este escenario, nos dicen, es muy improbable y esperamos que así sea. Pero aquello que los consumidores señalan -demostrando gran sentido común- es que todas las nuevas tecnologías han tenido alguna seria desventaja. Mientras no sepamos lo que eso significa para los alimentos o procesos GM, en el equilibrio de probabilidades, algo va a salir mal. Lo más probable es que resulte ser un problema más bien ambiental que humano

vi La postura de línea dura contra los consumidores por parte de los Estados Unidos se ha reiterado últimamente. La moción para aprobar la vía rápida para un acuerdo FTA A utiliza un lenguaje que tiende a establecer como objetivos de las negociaciones que el etiquetado de los OGM representa una barrera para el comercio. 
pero, de cualquier modo, los consumidores quieren poder "votar" con su dinero en el mercado si apoyan o no a la tecnología. Tienen el derecho a proceder así en un mercado libre, cualquiera sea la razón en que basan su elección.

Hay otros motivos de preocupación. La firma biotecnológica Monsanto advirtió al Departamento Británico del Medioambiente en 1999 que, accidentalmente, colocó dos fragmentos extra de genes en el poroto de soya Roundup Ready. En tanto que los fragmentos se encuentren inertes, no parecen constituir ningún riesgo. Sin embargo, esto da cuenta acerca del hecho que esta tecnología es menos comprensible de lo que muchos científicos y firmas biotecnológicas nos tratan de hacer creer.

El descubrimiento de los fragmentos genéticos ocho años después de la aprobación de esas semillas, nos lleva a cuestionar el aspecto impredecible de la tecnología. Y en febrero del 2000, las llamadas"super malezas" empezaron a aparecer. El departamento canadiense de agricultura confirmó que una semilla (parásita de la planta canola) había resultado ser resistente a tres clases de herbicida, habiendo tomado genes de tres diferentes variedades de semillas GM. Si las super malezas resultan ser un problema mayor para la agricultura, lo que no está tan claro aún, -aunque hay muchos científicos que están preocupados- lo que sí está claro es que la tecnología se encuentra en su infancia y muchas de sus características todavía no son comprendidas.

Debido a la incertidumbre respecto a los efectos de los cultivos GM en el medioambiente, muchos países están ahora estableciendo una moratoria o buscando acuerdos con las empresas de biotecnología para detener la producción de plantas genéticamente modificadas hasta que se hayan realizado pruebas más extensas. Nueva Zelandia y Alemania se han incluido recientemente en este grupo. ¿Podrían los GM convertirse en un problema serio para la salud? Absolutamente. ¿Podremos nosotros manejar eso? Eso es discutible. Como señaló claramente la Asociación Médica Británica, "sin separar los alimentos GM de los no GM y sin una clara etiquetación, la vigilancia rigurosa de la salud es imposible"vii.

Varios organismos reguladores estatales se han declarado enérgicamente a favor de la seguridad de los alimentos GM. Por ejemplo, lo dicho por la Autoridad Australiana y Neozelandesa de Alimentos (ANZFA) puede ser descrito "solamente" como atrevido: "toda la evidencia científica existente antes de la constitución de la ANZFA indica que los alimentos GM sometidos a análisis tienen todos los beneficios de los alimentos convencionales y no representan peligro adicional", iii. Los consumidores realmente esperan que esta autoridad tenga razón. Pero nótese el lenguaje cuidadosamente escogido: "toda la evidencia cientifica existente antes de..." Dado que no ha habido seguimiento de largo plazo para establecer la seguridad de los alimentos GM y que, además, los legisladores están permitiendo que los alimentos GM sean usados por los industriales sin la adecuada segregación o etiquetado - haciendo que la vigilancia médica rigurosa sea imposible, tal como lo ha señalado la profesión médica-, no resulta raro que los entes reguladores de alimentos sean atrevidos.

La Presidente de la Oficina de Investigaciones en Política de Salud de la Asociación Médica Británica, Dra. Vivienne Nathanson, ha expuesto claramente las preocupaciones de salud: "El hecho de que no haya evidencia de efectos adversos, no es lo mismo que la certidumbre y la confianza de que la modificación genética es segura. Por cierto, a menos que tengamos etiquetas adecuadas que segreguen los alimentos GM de los no GM,

vii British Medical Association, publicación de prensa: "BMA responde al jefe médico y a los representantes científicos". Revista de Alimentos GM y Salud 21/5/99.

viii Autoridad Alimentaria deAustralia y Nueva Zelandia, publicación de prensa: ANZFA anuncia protecciones de seguridad para alimentos GM para conocimiento público, 19/6/2000. 
nunca podremos establecer una seguridad satisfactoria. La mirada retrospectiva es quizá la única ciencia exacta y no acepto que nada en la vida esté exento de riesgo. Pero necesitamos saber más sobre los riesgos y las ventajas antes de aceptar esta nueva tecnología de alimentos" $" i x$.

La preocupación por los alimentos GM es tan grande que, en el Congreso Mundial en Sud África en noviembre del 2001, Consumers International adoptó la siguiente posición, reconociendo que la biotecnología y otras tecnologías de alimentos pueden traer beneficios importantes “... los gobiernos deben requerir una completa evaluación previa a su comercialización y un análisis del impacto social y de la seguridad de los alimentos genéticamente modificados y de aquéllos producidos por otras tecnologías alimentarias, para asegurar que sean seguras, ambientalmente sustentables y aceptables para los consumidores/as, e imponer una moratoria al cultivo y a la comercialización de los alimentos genéticamente modificados hasta que lo anterior se verifique"( 1).

\section{Antimicrobianos}

Un último caso para ilustrar por qué la precaución es tan necesaria en la legislación sobre alimentos se refiere al creciente problema de la resistencia a los antibióticos en humanos. Como lo ha establecido la Organización Mundial de la Salud “ desde su descubrimiento, los antibióticos han transformado completamente la situación de la humanidad respecto a las enfermedades infecciosas" (2). El riesgo para la salud humana proveniente de la resistencia a los antibióticos es una de las mayores amenazas emergentes para la salud en el siglo XXI. Aunque la resistencia antimicrobiana es un fenómeno biológico natu-

ix British Medical Association, publicación de prensa: "BMA responde al jefe médico y a los representantes científicos". Revista de Alimentos GMy Salud 21/5/99. ral, el uso de antimicrobianos para criar animales comestibles (opuesto al uso terapéutico responsable en animales) está exacerbando el problema de resistencia a las drogas.

De los antibióticos producidos hoy día, sólo la mitad son para uso en humanos (2, p.10), la otra mitad se usa en la industria ganadera, la mayor parte en promover el crecimiento y usos profilácticos en animales destinados al consumo humano. Recientemente, hay preocupación por el surgimiento de cepas no sólo resistentes, sino que, además, "saltan" de una especie huésped a otra. Este es el caso del enterococo resistente a la vancomicina (ERV) que apareció en animales y parece haber "saltado" a segmentos más vulnerables de la población humana. La ERV presente en los alimentos parece tener su origen en el uso de avoparcina en la ganadería; la avoparcina es el equivalente animal del antibiótico vancomicina que se usa en los humanos.

También existe gran preocupación a escala mundial acerca de la eficacia de los fluoroquilonoles (miembros del grupo quinolones de los antibióticos) y otros grupos de antibióticos. Hay un incremento en la aparición de agentes patógenos resistentes a los antibióticos, tanto en hospitales como en la comunidad. Básicamente, la medicina está perdiendo la principal línea de defensa.

La obvia actitud precautoria de parte de los gobiernos al respecto es asegurarse que los antibióticos que son usados para el tratamiento de infecciones en humanos no sean utilizados como agentes estimulantes del crecimiento en la ganadería. No es de sorprender que ésta sea la recomendación de sentido común que hace la Organización Mundial de la Salud, así como la mayoría de los gremios médicos y la mayor parte de los grupos organizados de consumidores. En tanto este principio de precaución ha sido observado en cierta medida en muchos países, su implementación es inconsistente y la verdad es que algunos países están haciendo muy poco. Aparentemente, la industria y sus intereses económicos han 
predominado sobre el derecho a la salud de los ciudadanos en muchos casos y en el excesivo tiempo que se le ha dado a la industria ganadera para ir reduciendo las dosis de antibióticos. Una vez que un "bicho" ha mutado en un país, es muy posible que, rápidamente, se extienda a otros.

Como en los casos citados anteriormente, la postura de los gobiernos debe ser claramente la de mantener un alto nivel de precaución mientras la investigación científica establece la dimensión del problema y el permiso futuro del uso de antibióticos en la ganadería. La precaución debiera, probablemente, generar una rápida suspensión del uso de antibióticos como estimulantes de crecimiento del ganado junto con alguna asistencia financiera hacia los productores durante la transición con el fin de impedir un mayor deterioro en la eficacia los de antibióticos. Aunque no se usen antibióticos en animales, existe un gran problema con el uso prudente de esas drogas en humanos; este peligro no debe ser exacerbado. Para muchos consumidores, la precaución tardará mucho en llegar a ponerse en vigor $y$, para la medicina, la capacidad de manejar ciertas enfermedades puede terminar sufriendo un gran retroceso.

\section{Seguridad alimentaria: sentido común de la buena política y de la buena economía}

En el ámbito de los principios, es muy fácil argumentar lo que es una buena política pública y asegurar una oferta alimentaria segura que no plantee una amenaza para el medioambiente. Es, por supuesto, más difícil aplicar estos principios a las decisiones específicas que necesitan ser tomadas. Pero hay buenas razones — no sólo de carácter éticopara asegurar el resultado precautorio. Es un buen sentido económico tener fuertes regulaciones que protejan a los consumidores.

En su trabajo germinal La ventaja competitiva de las naciones (3) Michael Porter demostró que una legislación permisiva y una pobre política de protección medioambiental no conducen al éxito económico de un país, de hecho acontece todo lo contrario. Algunas citas a continuación contienen lo esencial de su posición: "El gobierno altera las condiciones de la demanda a través de regulaciones que afectan las normas de los productos y de los procesos mediante los cuales dichos productos son fabricados, con pautas de rendimiento de calidad, de seguridad e impacto ambiental... Estándares restrictivos para los productos, con seguridad y control del impacto ambiental, contribuyen a mejorar la ventaja competitiva. Presionan a las empresas para mejorar la calidad, actualizar la tecnología y las distinguen en áreas de interés para los clientes y para las preocupaciones sociales... Resultan particularmente benéficos unos estándares restrictivos que anticipan los estándares que serán aplicados en el mundo entero. Esto les procura a las empresas nacionales una ventaja en el desarrollo de productos y servicios que serán valorizados en otra parte...Las empresas, como los gobiernos, frecuentemente se inclinan a ver los costos en el corto plazo en vez de confrontarse con normas estrictas, y no ven los beneficios a largo plazo en función de la innovación... Las empresas alegan que la competencia extranjera, no sometida a los mismos estándares rigurosos, tiene una ventaja de costo. Esa manera de pensar se basa en una visión parcial de cómo se crea y se sostiene la ventaja competitiva. Vender productos de bajo rendimiento, de funcionamiento inseguro o que dañan el medioambiente no es una buena ruta para obtener ventajas competitivas... Las regulaciones minan la ventaja competitiva, sin embargo, si la legislación de una nación se queda atrás respecto a las otras, son anacrónicas, tales regulaciones van a retardar la innovación o canalizar la innovación de las empresas locales en la dirección equivocada".

La mayor parte de la obra de Michael Porter es una empírica evidencia sobre sus teorías. Las citas anteriores, en la medida que se refieren a varios aspectos, implican dimensiones muy importantes para los países en vías de desarrollo, sobre todo en lo que se 
refiere al debate sobre su postura competitiva en el contexto global. Estos asuntos, a su vez, hacen surgir la cuestión del debate ético acerca de mejorar la transferencia tecnológica de las naciones desarrolladas a las menos pudientes.

En términos generales, muchos de los compromisos suscritos por los gobiernos, a todos los niveles, en sus decisiones acerca de la seguridad alimentaria han sido motivados por el interés de crear nuevos empleos o mantenerlos. Uno de los atractivos de dichas decisiones ha sido el de tener "menos restricciones". Esos compromisos que han sido adquiridos no son inmateriales. Muchos de ellos han desembocado en una disminución de la protección al consumidor y en una mengua de su bienestar. En algunos casos, como el de la ideología de auto-regulación de algunos estados conservadores, el "gobierno ausente" ha llevado a la muerte de ciudadanos ${ }^{\mathrm{x}}$. El análisis de Michael Porter desafía esa actitud de los gobiernos no al nivel ético, sino al nivel económico fundamental; de hecho, argumenta, que una postura de "ninguna o poca regulación" eventualmente le va a costar a un país muy caro en términos de atraer inversión y empleos porque las exportaciones del país no van a satisfacer los estándares que los consumidores desean.

En el ámbito internacional, los compromisos en seguridad alimentaria en la legislación muchas veces derivan de los poderosos intereses de los países que representan a sus sectores comerciales en detrimento del interés del ciudadano común. En esto, una vez más, la conducta de los gobiernos ha variado considerablemente. El hecho es que cuando un país poderoso, en una negociación internacional sobre normas de alimentos adopta una postura anti-consumidor, la agencia internacional se encuentra con que es muy difícil manejar el asunto ya que la mayor parte del proceso implica consenso.

Un gobierno adopta una mala posición política cuando opta por proteger a su industria, en lugar de sus ciudadanos, cuando la seguridad está en riesgo. En algún momento, los agentes de gobierno cuyas prioridades están en conflicto o son poco claras, cometerán un error fundamental de juicio. El más sorprendente de esos errores fue el de la vaca loca (BSE) y ha habido muchos otros. Fallarles así, tan seriamente, a los consumidores, significará en último caso que la industria misma será penalizada. Uno sólo necesita mirar el número de consumidores que no comen carne de res en el Reino Unido, en Europa y otros países, para ver cuánto daño puede sufrir un sector industrial cuando los gobiernos dejan de actuar apropiadamente.

Si los consumidores pierden confianza en la seguridad de sus alimentos, no sólo la industria va a sufrir, también los gobiernos pueden caer por estas causas.

\footnotetext{
x Hay muchos ejemplos que podría uno encontrar en casi todos los países. Para dar un ejemplo australiano, un organismo de gobierno desreguló substancialmente la inspección de alimentos y traspasó la responsabilidad a la propia industria. Se produjo un brote de intoxicación alimenticia a través de una especie de salame que dejó unos 100 enfermos y, al menos, una víctima fatal infantil. Ese organismo gubernamental todavía alega, ahora con menos énfasis, que la autoregulación debiera funcionar.
} 
Acta Bioethica 2001; año VII, nº 2

\section{Referencias}

1. Consumers International. Consumidores, justicia social y el mercado mundial: Declaración del 16th Congreso Mundial de Consumers International. Noviembre 2000. [On Line ]. Available from:URL: www.consumerinternational.org

2. World Health Organization. Informe mundial de salud sobre enfermedades infecciosas 2000. Superando la resistencia antimicrobiana. Ginebra: WHO; 2001. [On Line]. Available from: URL: www.who.int/infectious-disease-report/2000

3. Porter M. The competitive advantage. New York: Macmillian Press; 1990. 